\title{
Multi-Agent Reinforcement Learning based Distributed Renewable Energy Matching for Datacenters
}

\author{
Haoyu Wang, Haiying Shen, Jiechao Gao, Kevin Zheng, Xiaoying Li \\ University of Virginia \\ Charlottesville, Virginia, USA \\ \{hw8c, hs6ms, jg5ycn, ksz3kd, xl2gs\}@virginia.edu
}

\begin{abstract}
The rapid growth of cloud computing in cloud datacenters in recent decades greatly increases the brown energy consumption in datacenters, and hence significant increase of carbon emission that negatively impacts on the environment as well as the monetary cost. More and more cloud service providers are adopting renewable energy as the energy supply to offset the consumption of brown energy. Meanwhile, an increasing number of renewable energy generators have been built to meet the needs. However, the instability of the renewable energy cannot guarantee the support to the datacenter and the energy competition of different datacenters may lead to datacenter energy outage. In this paper, we focus on the problem of how to match different renewable energy generators to the datacenters from different cloud providers to minimize the carbon emission, monetary cost, and service level objective (SLO) violation due to renewable energy shortage. The challenges here are that the datacenters may compete in energy requesting, the renewable energy generation is not stable and the decision should be made quickly. There have been no previous efforts devoting to this problem. To solve the problem, we first test several machine learning techniques on long-term prediction accuracy on renewable energy generation and energy demand using real traces and identify SARIMA for the prediction. We then propose a multi-agent reinforcement learning based method (MARL) for each datacenter to determine how much renewable energy to request from each generator based on the predicted results. We also propose a deadline guaranteed job postponement method (DGJP) to postpone executing unurgent jobs upon insufficient renewable energy supply. The trace-driven experiments show that MARL outperforms other methods by increasing up to $35 \%$ SLO satisfaction ratio, and reducing up to $19 \%$ ( 0.33 billion dollars in 90 days) total monetary cost and $33 \%$ total carbon emission, and DGJP further improves the performance.
\end{abstract}

\section{ACM Reference Format:}

Haoyu Wang, Haiying Shen, Jiechao Gao, Kevin Zheng, Xiaoying Li. 2021. Multi-Agent Reinforcement Learning based Distributed Renewable Energy Matching for Datacenters. In 50th International Conference on Parallel Processing (ICPP '21), August 9-12, 2021, Lemont, IL, USA. ACM, New York, NY, USA, 10 pages. https://doi.org/10.1145/3472456.3473514

Permission to make digital or hard copies of all or part of this work for personal or classroom use is granted without fee provided that copies are not made or distributed for profit or commercial advantage and that copies bear this notice and the full citation on the first page. Copyrights for components of this work owned by others than ACM must be honored. Abstracting with credit is permitted. To copy otherwise, or republish, to post on servers or to redistribute to lists, requires prior specific permission and/or a fee. Request permissions from permissions@acm.org.

ICPP '21, August 9-12, 2021, Lemont, IL, USA

(C) 2021 Association for Computing Machinery.

ACM ISBN 978-1-4503-9068-2/21/08...\$15.00

https://doi.org/10.1145/3472456.3473514

\section{INTRODUCTION}

Geo-distributed datacenters provide global cloud services and such a datacenter requires an enormous amount of energy (megawatts of electricity) which led to the cloud service provider companies, such as Google, Microsoft and Amazon, spend tens of millions of dollars annually for electricity [1, 35, 49]. The estimation in 2018 shows that the worldwide datacenters consume around $1 \%$ of the total electricity used worldwide [11]. A large number of datacenters around the world are powered by brown energy such as fossil fuel, coal, and oil, which generate a large amount of carbon emission. Indeed, the total volume of carbon emission will be 1430 million metric tons, and the datacenters is responsible for $18 \%$ carbon emission in the world by $2020[47,50]$. The carbon emissions raise global temperatures, alter water supplies and weather patterns, change the growing season for food crops, and threaten coastal communities with increasing sea levels [4].

In order to mitigate the adverse environmental impact and the energy crisis caused by the rapidly increased brown energy consumption, many companies (e.g., Google, Microsoft and Amazon) have started to power their datacenters using the renewable energy as much as possible [2, 3, 7, 27]. For example. Apple built a $40 \mathrm{MW}$ solar array for its North Carolina datacenter [22]. However, a datacenter cannot always use the renewable energy generators to satisfy its energy demand because of the variant amount of renewable energy from renewable energy generators[42, 43, 45]. Even when using the brown energy supply, a common cause of datacenter failure is power outages such as those documented by Yahoo! [39], LinkedIn [17] and Facebook [15]. Since the CPU of a machine cannot be fully utilized with insufficient energy supply [33] and it takes a while to switch to the brown energy supply upon renewable energy shortage, the jobs on this machine cannot be executed with full speed and then the job completion time will be increased. Therefore, the shortage of renewable energy supply may lead to the violation of the job's service level objective (SLO) such as the percentage of the requests or jobs from a customer that can be served or completed before the job deadlines. As a result, it is important for a datacenter to determine the amount of renewable energy to purchase to avoid renewable energy shortage as well as overpurchase. Suppose that in a large area, there are many datacenters belonging to different cloud providers and many renewable energy generators; each datacenter purchasing renewable energy from one or multiple energy generators, and a generator distributes the generated renewable energy to its requesters. In this scenario, it is important for each datacenter to determine how much renewable energy to request from each generator in order to receive sufficient energy to support its job SLOs while minimizing 
the monetary cost and carbon emission. Specifically, this renewable energy demand-supply matching problem can be described as below:

Given a set of datacenters belonging to different cloud providers and many renewable energy generators (with each generator generating one type of energy) geo-distributed in an area, how should the renewable energy generators be matched to different datacenters in order to fulfill their energy demands in a long term (e.g., one month) to minimize application SLO violation rate (due to interruption from insufficient renewable energy), total carbon emission and monetary cost of each datacenter?

This problem has not been investigated in previous research. Previous works for renewable energy demand-supply matching problem focus on multiple datacenters belonging to the same cloud provider [24, 36]. Then, the datacenters' information can be obtained by a centralized agent and there is no demand competition among datacenters, which cannot be applied to our problem. Also, these methods only achieve a short-term (e.g., one hour) generatordatacenter matching plan resulting in frequent plan changes and hence extra overhead [6].

In this paper, we aim to solve the above problem and achieve long-term planning to avoid extra overhead. To achieve long-term planning, we use several machine learning (ML) algorithms (i.e., SVM (support vector machine), LSTM (long short term memory), and SARIMA (seasonal-ARIMA)) to do the energy generation and demand prediction using over five years of energy generation and demand data. We then select SARIMA that produces the highest prediction accuracy to make the long-term prediction on demand/supply at each time slot (e.g., each hour) in a long term (e.g., the next month). In addition, since multi-agent reinforcement learning can handle the competition between agents to achieve global optimum without the communication between the agents, we propose a multi-agent RL based method (MARL). One RL agent deployed on each datacenter makes the decisions individually on how much energy is requested from each energy generator. We sum up our contributions as follows:

(1) We compared the prediction accuracies of several ML techniques using real datasets and chose SARIMA that can achieve the highest accuracy. Each datacenter uses SARIMA to predict the amount of generated renewable energy in each renewable energy generator and its energy demand at each time slot in the next month for its decision making.

(2) Based on the predicted renewable energy supply and energy demand, each datacenter uses MARL to determine how much renewable energy to request from each generator to achieve the goals in the problem above.

(3) We propose a Deadline-Guaranteed Job Postponement method (DGJP). When there is a shortage of renewable energy supply, DGJP postpones the execution of the jobs with later deadline requirements to a time period with a higher amount of energy supply than the demand.

(4) We conduct comprehensive real trace-driven experiments to compare our method with other three methods in terms of SLO satisfactory ratio, total energy monetary cost and total carbon emission in a long time period. The experimental results show that our method can achieve a much lower SLO violation ratio, total energy monetary cost, and total carbon emission compared to the other methods.

\begin{tabular}{|l|l|l|l|l|l|l|}
\hline Work & $\begin{array}{l}\text { Multi } \\
\text { datacenters }\end{array}$ & $\begin{array}{l}\text { Constrained } \\
\text { by fixed } \\
\text { matching }\end{array}$ & $\begin{array}{l}\text { Carbon } \\
\text { emission }\end{array}$ & $\begin{array}{l}\text { Monetary } \\
\text { cost }\end{array}$ & SLO & Multi CSP \\
\hline Cplex [16] & $\checkmark$ & $X$ & $\checkmark$ & $X$ & $\checkmark$ & $X$ \\
\hline REA [17] & $\checkmark$ & $X$ & $\checkmark$ & $X$ & $X$ & X \\
\hline WST [18] & $\checkmark$ & $X$ & $\checkmark$ & $X$ & $X$ & X \\
\hline TM [19] & $X$ & $X$ & $\checkmark$ & $X$ & $X$ & $X$ \\
\hline REM [8] & $X$ & $X$ & $\checkmark$ & $\checkmark$ & $\checkmark$ & $X$ \\
\hline GS [20] & $X$ & $X$ & $\checkmark$ & $X$ & $\checkmark$ & $X$ \\
\hline FF_LPT [21] & $X$ & $X$ & $\checkmark$ & $\checkmark$ & $X$ & $X$ \\
\hline Linear [13] & $\checkmark$ & $\checkmark$ & $X$ & $\checkmark$ & $\checkmark$ & $X$ \\
\hline OPT [14] & $\checkmark$ & $\checkmark$ & $\checkmark$ & $\checkmark$ & $X$ & $X$ \\
\hline SRL [42] & $X$ & $\checkmark$ & $\checkmark$ & $\checkmark$ & $\checkmark$ & $X$ \\
\hline Our work & $\checkmark$ & $\checkmark$ & $\checkmark$ & $\checkmark$ & $\checkmark$ & $\checkmark$ \\
\hline
\end{tabular}

Figure 1: Comparison of related work.

There are other approaches for handling the shortage of renewable energy supply such as storing renewable energy for future use. Our methods can be complementary to those approaches to strengthen the capability to handle the energy shortage. The rest of the paper is organized as follows. Section 2 presents the related work. Section 3 presents the system design. Section 4 presents the performance evaluation of our system. Section 5 concludes the paper with remarks on our future work.

\section{RELATED WORK}

The problem of using renewable energy to support datacenters has been investigated in recent years. We classify the previous research works into the following three categories. Figure 1 shows the comparison table of the related work.

Job scheduling among multiple datacenters. Some works focus on a problem that given a set of jobs, multiple datacenters belonging to one cloud service provider, how to determine which datacenter to run each job for certain objectives. Gu et al. [23] proposed an integer linear programming method aiming to minimize the total carbon emissions of all the datacenters under electricity monetary budget and job processing time constraint. Xu et al. [48] proposed an RL based job scheduling algorithm aiming to minimize the energy consumption. The RL method can achieve the near-optimal solutions by learning the historical job scheduling decision. This method tends to migrate jobs from the datacenters without enough renewable energy supply to the datacenter with more renewable energy supply so that renewable energy can be utilized as much as possible.

Job scheduling in a datacenter. Some works focus on a problem that given a set of jobs on one datacenter, how to determine when to run each job for certain objectives. Li et al. [29] proposed a heuristic based job scheduler aiming to maximize the renewable energy utilization and reduce the usage of brown energy based on LSTM prediction on generated energy amount. Goiri et al. [22] proposed a job scheduler, GreenSlot, aiming to maximize the usage of renewable energy and minimize the total monetary cost while meeting the jobs' deadlines. GreenSlot predicts the amount of renewable energy generation, and then schedules the jobs at the time when there is enough energy supply. Liu et al. [32] proposed a job scheduling method aiming to minimize the carbon emission while meeting jobs' deadlines. This method first predicts the generated amount of renewable energy using the Fast Fourier Transform 


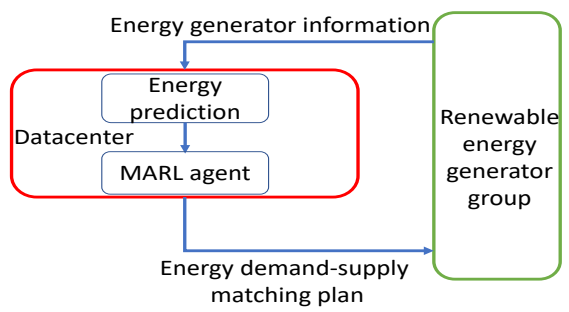

Figure 2: Overview of MARL based system.

(FFT) technique and then tries to schedule more jobs to run when the predicted amount of renewable energy supply is higher. De Courchelle et al. [20] proposed a job scheduling method aiming to use renewable energy as much as possible while minimizing the monetary cost and meeting jobs' deadlines. This method first predicts the generated amount of renewable energy including solar energy and wind energy based on weather forecast, and then prefers to schedule the jobs when there is enough renewable energy to support this datacenter; otherwise it just delays the jobs until when the renewable energy supply is enough. However, the above job scheduling based methods assume that the energy generators supplying energy to a given datacenter are fixed, so these methods cannot be applied to our problem in this paper.

Energy demand-supply matching. Some other works focus on a problem that given multiple datacenters belonging to one cloud service provider and multiple energy generators (which have different prices according to different locations and different times), how to determine which generators are used to power each datacenter for certain objectives. Rao et al. [36] proposed a constrained mixed-integer programming method aiming to minimize the total energy monetary cost while guaranteeing that the jobs' deadline satisfaction rate for all the datacenters. Gu et al. [24] considered the case that all the edge nodes and energy generators are belonged to a single cloud service provider. The authors proposed a mixed integer linear programming based algorithm aiming to use renewable energy as much as possible, minimize total monetary cost for brown energy and fulfill the energy demand of each datacenter. Since different jobs have different energy demands with time diversity and different edge nodes have different computing ability with different energy consumption, this method first allocates different jobs to different edge nodes aiming to minimize the energy consumption. After the job allocation, since the energy transmission between edge node and renewable energy generator causes energy loss, which is related to their distance, this method then schedules different renewable energy generators to different edge nodes aiming to minimize the total energy loss.

Unlike these works, we focus on the scenario that the datacenters do not belong to the same cloud service provider. Then, the competition among datacenters may lead to energy shortage of several datacenters, which prevent them from achieving the goal in the above stated problem.

\section{SYSTEM DESIGN FOR ENERGY DEMAND-SUPPLY MATCHING}

In this section, we propose a MARL based demand-supply matching algorithm, which aims to generate a matching plan between energy generators and datacenters in order to always fulfill each

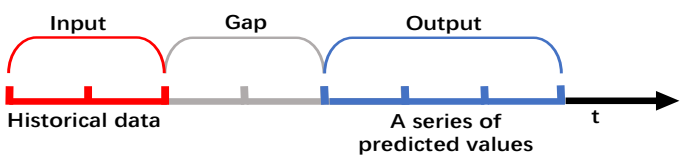

Figure 3: Prediction inputs and output in our prediction.

datacenter's energy requirements using renewable energy as much as possible, minimize SLO violation caused by lack of energy, carbon emission and money cost. Note that the owner of a datacenter can flexibly set the weights of these goals or even modify the goals by changing MARL's reward function. In the following, we first present a method for a datacenter to predict its own energy demand and the amount of the generated renewable energy of each renewable energy generator at each time slot in the next month. The predicted amounts will be used in the MARL based algorithm. Second, we formulate the energy generator and datacenter matching problem into a Markov game [31]. The Markov game includes the traditional Markov problem formulation and also game theory which considers the competition and cooperation among all the players (datacenters) in the game. Third, we present the MARL algorithm to solve this problem. Fourth, we present our proposed DGJP method for further enhancement. Figure 2 shows the structure of the MARL based system.

\subsection{Energy Demand and Supply Prediction}

Previous work [36] predicts the demand and supply amounts at a single time slot each hour and conducts the energy demandsupply matching hourly. First, this would lead to frequent (hourly) matching plan changes and generate extra overhead. Second, the prediction is conducted one hour before the ready-to-predict time slot. Considering the potential ML prediction algorithm time overhead, it may not leave enough time to use the predicted values to compute the matching plan and execute the plan, which are time consuming [19].

To handle the first problem, our prediction method predicts a series of amounts for each hour in the next month to achieve the long-term prediction. To handle the second problem, we propose to leave a sufficient time gap between the time slot doing prediction and the ready-to-predict time slot. For example, in the previous research, the algorithm only predicts $5 \mathrm{kWh}$ at $5 \mathrm{PM}$, Monday. Our prediction method predicts a series of energy values for a time period such as (4kWh, 5PM), (4.2kWh, 6PM), (4.8kWh, 7PM),... As shown in Figure 3, our prediction method inserts a gap (e.g., one month) period between the input data points and the first output data point. The input data is the historical time-series data of the amount of a generator's generated energy or a datacenter's demand and the output data is a series of corresponding predicted values. Both energy generation prediction and energy demand prediction will use this prediction method to make sure there is enough time left for the datacenters to make datacenter-generator matching plans and also the execution of this plan. Also, the series of predicted energy values can help make a long-term energy matching plan and thus avoid frequent matching plan changes. Take predicting generated energy for generator $G_{k}$ for $Z$ time slots as an example, the prediction is represented by:

$$
\mathcal{D}_{G_{k}}=\left\{\mathcal{D}_{G_{k}, t_{1}}, \mathcal{D}_{G_{k}, t_{2}}, \ldots, \mathcal{D}_{G_{k}, t_{z}}, \ldots, \mathcal{D}_{G_{k}, t_{Z}}\right\},
$$



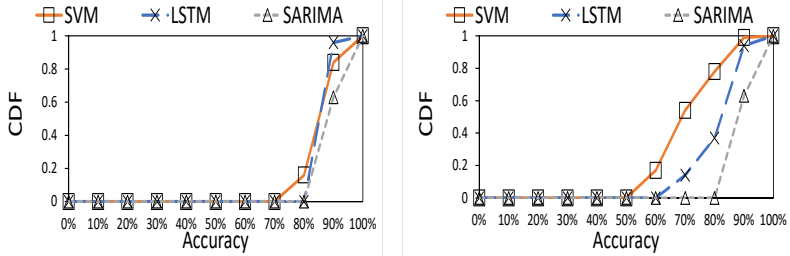

Figure 4: Solar renewable en-Figure 5: Wind renewable energy prediction. ergy prediction.

in which

$$
\mathcal{D}_{G_{k}, t_{z}}=\left\{\left(v_{1}, p_{1}\right),\left(v_{2}, p_{2}\right), \ldots,\left(v_{i}, p_{i}\right), \ldots,\left(v_{m}, p_{m}\right)\right\}
$$

where $m$ is the total number of possible value for the energy generator, and $v_{i}$ and $p_{i}$ denote the predicted value and the probability that the predicted value equals to $v_{i}$. We define the predicted energy demand for datacenter $D_{k}$ in the same way.

As the time-series datasets of multiple generators share certain features but the generators still have different attributes such as location, scale and weather, a datacenter builds one model for predicting the generated amount of each generator (to leverage the different datasets for high accuracy) and uses the generator ID as an input (to differentiate the generators). We assume that each generator will publicize its generated amount for its customers to make plans. If this data of a generator is not available, a datacenter can conduct the prediction based on weather data (e.g., wind speed and solar density) as we do in the following.

The next question is which ML technique we should choose that can achieve high prediction accuracy. We choose SVM [38], LSTM [25] and SARIMA [18], which are widely used in time-series data prediction, to compare their prediction accuracy performance. Since SVM cannot predict a series of data points one time, we run SVM once for each predicted time slot. To predict datacenter energy demand, we use the Wikipedia trace dataset [14]. The trace data includes hourly number of requests to each web page (30 million in total) of Wikipedia from November 30, 2011 to December 1, 2016 for five years. We use the number of requests to evaluate the CPU utilization and then transform it to energy consumption using the approach introduced in [28] since CPU utilization is a good estimator for energy consumption. We use one month (e.g., March) hourly time-series points and its corresponding energy consumption values as the input and the model outputs the energy consumption values at each hour in the next second month (e.g., May) with a gap of one month in between.

For predicting the amount of generated renewable energy of a generator, we use the time-series solar irradiance dataset from [9] and time-series wind speed dataset from [10] as the energy examples and calculate the amount of renewable energy generation according to the methods in [37] and [40], respectively. The trace datasets include hourly values at Virginia, Arizona and California states from December 1, 2011 to November 30, 2016 for five years. We use one month hourly time-series points of produced energy (e.g., 720 points in 30 days) as input and the model outputs the corresponding renewable energy values (e.g., 720 values) in the next second month with a gap of one month in between. We use the first three years' data as the training data and the rest as the test data.
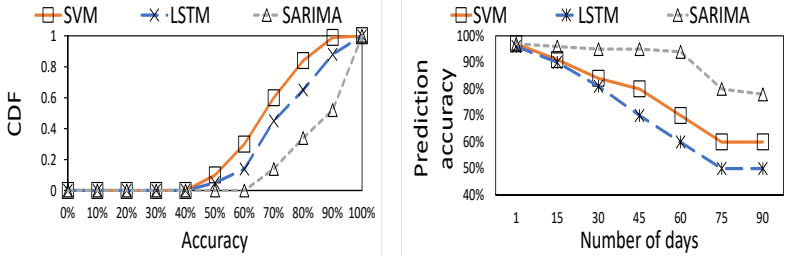

Figure 6: The prediction of Figure 7: Prediction accuracy energy demand. with different gaps.

We calculate the prediction accuracy by: $A_{n}=1-\frac{P_{n}-R_{n}}{R_{n}}$ where $A_{n}$ is the corresponding prediction accuracy for the $n^{t h}$ predicted value, $P_{n}$ is the $n^{t h}$ predicted value and $R_{n}$ is the $n^{t h}$ real value. Figure 4 and Figure 5 show the cumulative distribution function (CDF) of the prediction accuracy of the wind energy prediction and solar energy prediction, respectively, using SVM, LSTM and SARIMA. We see that SARIMA achieves the highest prediction accuracy. LSTM and SARIMA are good at handling time-series data than SVM in predicting time-series data. Also, SARIMA can better catch the seasonal pattern for the time series data for the overall time period than LSTM when conducting the prediction. Figure 6 shows the CDF of the prediction accuracy of the energy demand using SVM, LSTM and SARIMA. We can see SARIMA achieves the highest accuracy due to the aforementioned reason.

Figure 7 shows the average prediction accuracy of energy demand prediction with different time-length gaps. We see that ARIMA still achieves the highest accuracy. Meanwhile, with the increase of "gap", the prediction accuracy decreases for all method. The prediction accuracy of SARIMA is more stable than other two prediction methods. SARIMA can achieve stable prediction accuracy over $90 \%$ within 60 days of "gap". Therefore, we use SARIMA as our energy demand and energy generation prediction model.

\subsection{Problem Formulation}

In our problem, as the datacenters belong to different cloud service providers, it is infeasible to set up a centralized controller to obtain the information of all the datacenters and make the datacentergenerator matching plan globally. Considering that the datacenters are not cooperative and may compete in requesting the energy, it is practical to let each datacenter make the energy requesting decision by itself in a distributed manner. We formulate the datacentergenerator matching problem in Section 1 using Markov game [30]. Markov game is an extension of game theory to Markov Decision Problem (MDP) with additional consideration of the competition between agents to let each agent make the optimal decision.

3.2.1 Overall Definition. The Markov game is defined by a tuple $\left(\mathcal{N}, \mathcal{S}^{i}{ }_{i \in \mathcal{N}}, \mathcal{A}^{i}{ }_{i \in \mathcal{N}}, \mathcal{P}, \mathcal{R}^{i}{ }_{i \in \mathcal{N}}, \gamma\right) . \mathcal{N}=\left\{n_{1}, n_{2} \ldots, n_{N}\right\}$ denotes the set of agents in the Markov Game. $\mathcal{S}^{i}$ denotes the state space of the $i^{t h}$ agent and $\mathcal{S}:=\mathcal{S}^{1} \times, \ldots \times \mathcal{S}^{N}$ denotes the state space observed by all the agents. $s \in \mathcal{S}\left(s=\left\{s^{1}, \ldots, s^{i}, \ldots, s^{N}\right\}\right)$ denotes the joint observed state and $s^{i}$ denotes the state observed by the $i^{t h}$ agent. $\mathcal{A}^{i}$ denotes the action space of the $i^{t h}$ agent and $\mathcal{A}:=\mathcal{A}^{1} \times \ldots \times \mathcal{A}^{N} . a \in \mathcal{A}$ $\left(a=\left\{a^{1}, \ldots, a^{i}, \ldots, a^{N}\right\}\right)$ denotes the joint action and $a^{i}$ denotes the action taken by the $i^{t h}$ agent. $\mathcal{P}: \mathcal{S} \times \mathcal{A} \rightarrow \Delta(\mathcal{S})$ denotes the transition probability from a state $s \in \mathcal{S}$ for an action $a \in \mathcal{A}$. For 
$\mathcal{R}^{i}{ }_{i \in \mathcal{N}, \gamma}, \mathcal{R}^{i}: \mathcal{S} * \mathcal{A} \rightarrow \mathbb{R}$ is the reward function that determines the immediate reward received by agent $n_{i}$ for a transition from $s$ to $s^{\prime}$ by taking $a$. We use $r^{i}$ to denote the reward calculated by the $\mathcal{R}^{i}$ function. $\gamma(0<\gamma<1)$ is the discount factor which controls how much effect future rewards have on the optimal decisions [31]; a smaller $\gamma$ gives a high weight to the recent gain reward and a larger $\gamma$ gives a higher weight to the future reward. The goal of each agent in this Markov game is to maximize its expected sum of discounted rewards. In the following, we introduce the tuple element in our scenario.

3.2.2 State Space. The state space for the $i^{t h}$ agent $\mathcal{S}^{i}$ including the information of all the renewable energy generators and the datacenter of the agent. First, we denote the predicted generated energy amount for $t_{Z}$ time slots for the $k^{t h}$ renewable energy generator by:

$$
\mathcal{G}_{G_{k}}=\left\{g_{G_{k}, t_{1}}, g_{G_{k}, t_{2}}, \ldots, g_{G_{k}, t_{z}}, \ldots g_{G_{k}, t_{Z}}\right\}
$$

where $g_{G_{k}}, t_{z}$ means the amount of renewable energy generated by the $k^{t h}$ renewable energy generator. In addition, the unit price vector indicates the unit prices of an energy generator at different times:

$$
u_{G_{k}}=\left\{u_{G_{k}, t_{1}}, u_{G_{k}, t_{2}}, \ldots, u_{G_{k}}, t_{z}, \ldots u_{G_{k}, t_{Z}}\right\},
$$

where $u_{G_{k}}, t_{z}$ denotes the energy unit price of generator $G_{k}$ in time slot $t_{z}$. The energy unit price is varied at different times and is pre-known for all the datacenters [5]. We define the predicted time-series energy demand vector of the $i^{\text {th }}$ datacenter:

$$
\mathcal{D}^{i}=\left\{d_{t_{1}}, d_{t_{2}}, \ldots, d_{t_{z}}, \ldots d_{t_{Z}}\right\},
$$

Then, $\mathcal{S}^{i}$ is expressed by:

$$
\mathcal{S}^{i}=\left\{\mathcal{D}^{i},\left(\mathcal{G}_{G_{1}}, u_{G_{1}}\right), \ldots,\left(\mathcal{G}_{G_{k}}, u_{G_{k}}\right), \ldots,\left(\mathcal{G}_{G_{K}}, u_{G_{K}}\right)\right\}
$$

where $K$ is the total number of renewable energy generators.

3.2.3 Action Space. The RL agent in a datacenter makes decisions only for this datacenter. Each action in the action space denotes a set of energy generator selected for each time slot in the next time period (e.g., one month). That is, how much energy should be requested from each energy generator. Note that a 0 request to a generator means that this generator is not selected. For the $i^{t h}$ datacenter, the action space for the $i^{t h}$ agent is represented as:

$$
\mathcal{A}^{i}=\left\{\mathcal{E}_{G_{1}}, \mathcal{E}_{G_{2}}, \ldots, \mathcal{E}_{G_{k}}, \ldots, \mathcal{E}_{G_{K}}\right\}
$$

where

$$
\mathcal{E}_{G_{k}}=\left\{\mathcal{E}_{G_{k}, t_{1}}, \mathcal{E}_{G_{k}, t_{2}}, \ldots, \mathcal{E}_{G_{k}, t_{z}}, \ldots, \mathcal{E}_{G_{k}, t_{Z}}\right\}
$$

where $\mathcal{E}_{G_{k}, t_{z}}$ means how much energy will be requested from energy generator $G_{k}$ within the time period $t_{z}$.

3.2.4 Probability. As described above, when an action is determined by a datacenter's agent about how much energy should be requested to each energy generator, the state is changed with certainty. Therefore, the probability between each state is always $1[44]$.
3.2.5 Reward. There are three parts in the reward function including monetary cost, carbon emission, and SLO violation caused by energy shortage. As noted before, the datacenter owner can modify the goals flexibly.

Monetary cost: Recall the unit price of energy generator $G_{k}$ at time slot $t_{z}$ is denoted by $u_{G_{k}}, t_{z}$. The monetary cost paid by the $i^{t h}$ datacenter for purchasing $\mathcal{E}_{G_{k}}, t_{z}$ amount of energy from energy generator $G_{k}$ at time slot $t_{z}$ is calculated by:

$$
\mathcal{C}_{G_{k}, t_{z}}^{i}=u_{G_{k}, t_{z}} \cdot \mathcal{E}_{G_{k}, t_{z}}+c \cdot b_{t_{z}},
$$

where $c$ is the monetary cost due to renewable energy generator switching and $b_{t_{z}}$ a binary variable indicating whether there is a generator switch in the time slot $t_{z}$.

Carbon emission: The amount of carbon emission per $\mathrm{kWh}$ of energy generator $G_{k}$ at time $t_{z}$ is denoted by $w_{G_{k}}, t_{z}$ which is calculated using the method in [8]. The total carbon emission for $\mathcal{E}_{G_{k}, t_{z}}$ amount of energy from energy generator $G_{k}$ at time $t_{z}$ is calculated by:

$$
W_{G_{k}, t_{z}}^{i}=w_{G_{k}, t_{z}} \cdot \mathcal{E}_{G_{k}, t_{z}} .
$$

SLO violation rate: Recall that the length of one time period is $Z$. Suppose the selected action in this time period is $a^{i}$, we use $V_{a^{i}, t_{z}}$ to denote the number of SLO violations in the time slot $t_{z}$ in the reward function. Therefore, the reward function during the time period $Z$ is:

$$
\mathcal{R}^{i}=\sum_{t_{z} \in Z} \sum_{k \in K} \frac{1}{\alpha_{1} * \mathcal{C}_{G_{k}, t_{z}}^{i}+\alpha_{2} * W_{G_{k}, t_{z}}^{i}+\alpha_{3} * V_{a^{i}, t_{z}}},
$$

where $\alpha_{1}, \alpha_{2}, \alpha_{3}$ are the weight coefficients for the different features respectively. These coefficients can be determined by the datacenter owner.

\subsection{Multiple Agent Reinforcement Learning based Algorithm}

Because of the unaccessible control of centralized controller, we use a distributed way to determine the energy-generation assignment plan by each datacenter itself. Meanwhile, since there is competition among those datacenters to compete for the energy generators, instead of single agent reinforcement learning, we propose an energy generation assignment based on multi-agent reinforcement learning (MARL) which considers the competition among all the datacenters. The MARL has been approved that it can succesfully to solve the agent competition problem and achieve better results $[26,41]$.

We use the MiniMax Q-learning algorithm [30] to build the MARL model to solve the formulated problem. Unlike the traditional Q-learning [46] in which the single agent determines its action with the largest $Q$ value based on its own information, in MiniMax, an agent considers the competitors' actions and then selects the action based on current state, which can maximize its reward under the worst case (with the assumption that all the competitors will take the actions to reduce its gain). That is, the MARL agent deployed on each datacenter can always generate the action to increase the possibility that the energy demand of this datacenter can be satisfied and the goals can be maximally achieved no matter what actions other datacenters take. As the Q-learning, the MiniMax Q-learning algorithm aims to find an optimal policy 
$\pi^{i}: s^{i} \rightarrow a^{i}$ (a function that maps the state to the optimal action). The Q-value function is used to estimate the expected reward for the given state-action pairs. The only difference between MiniMax Q-learning and Q-learning is the Q-value function.

MiniMax redefines the $Q$-value function as the expected reward for taking action when the competitors choose the actions from one state and continuing optimally thereafter. The MiniMax return value of a state $s^{i}$ is calculated by:

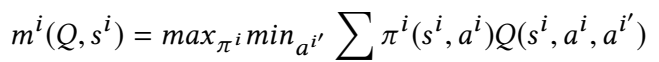

where $a^{i^{\prime}}$ means the actions from all the competitors of $i^{t h}$ agent. The Q-value function for the action $a^{i}$ in state $s^{i}$ against the competitors actions $a^{i^{\prime}}$ is:

$$
\begin{aligned}
& Q_{k+1}\left(s_{k}^{i}, a_{k}^{i}, a_{k}^{i^{\prime}}\right)=Q_{k}\left(s_{k}^{i}, a_{k}^{i}, a_{k}^{i^{\prime}}\right) \\
& \quad+\alpha\left[R_{k+1}^{i}+m_{i}\left(Q_{k}, s_{k+1}^{i}-Q_{k}\left(s_{k}^{i}, a_{k}^{i}, a_{k}^{i^{\prime}}\right)\right)\right]
\end{aligned}
$$

where $k$ is the iteration step for the $i^{t h}$ agent to update its Qvalue, $r_{k+1}^{i}$ is its received reward from the $k+1$ iteration based on Equation (11) and $\alpha$ is the learning rate which is used to guarantee the updated reward convergence.

In the training process of the MARL model, in the $k^{t h}$ iteration, after the $i^{t h}$ datacenter observes the sate $s_{k}^{i}$, it needs to take an action $a_{k}^{i}$ which determines how much energy should be requested from each energy generator (as in Equation (7)). The Q-values for different state-action pairs are stored in a Q-table, which indicate the corresponding cumulative reward achieved by an agent taking the action $\left(s_{k}^{i}, a_{k}^{i}\right)$ depending on the observed state $s_{k}^{i}$ and the action $a_{k}^{i}$. The agent takes the action with the highest Q-value. This action is selected only based on the current state. With the increase of the number of iterations, the agent keeps updating the Q-table according to Equation (13) based on the taken actions and the observed state $s_{k}^{i}$ and the calculated reward $r_{k}^{i}$. After many iterations, the MARL model converges and the MARL model training is completed. In this way, Q-value can be updated for minimizing the possible reward loss for the worst case scenario. The above recursive equations have been proved that it can finally converge to Nash Equilibrium state and then produce stable and near optimal solutions [16].

Next, we present the whole process of using our MARL-based method for the datacenter-generator matching problem. For the long-term prediction, the history running data (e.g., CPU utilization) stored in each datacenter is used to predict the energy demand in the next time period. Meanwhile, according to the history produced energy or weather data, each datacenter can predict the energy generation for each energy generator. An MARL agent is deployed in each datacenter and it takes the prediction results as input to make the datacenter-generator matching plan for the datacenter itself. If the predicted results are not correct (even with much low probability), the RL's actions based on the wrong prediction results lead to lower rewards in the training. The trained RL model can further reduce the negtive effect from the wrong predicted results. After the decision making, the reward will be calculated according to the information detected (e.g., energy cost and SLO violation rate) in the datacenter. The calculated reward will be used to train the MARL agent. This process repeats to update the agent model. The prediction and each agent run periodically in every month.
The datacenter will execute this datacenter-generator matching plan. The energy generator will then assign the requested energy amounts to the datacenter requesters.

Using MARL, the possibility that the total amount of energy requested from the datacenters is higher the amount of predicted generated energy is low. However, there is still a low possibility. In this case, the generator can use a certain policy to distribute the energy. For example, it can assign the amounts to the datacenters in proportion to their requested amounts. Also, the predicted generated energy amount may be higher than the actual amount due to weather change, e.g., hurricanes. In this case, a generator can also use the proportion policy to distribute the energy. When a new datacenter joins the system, it doesn't have the trained prediction model or the MARL model to use. Thus, the new datacenter needs to run using an existing renewable energy supply strategy (the datacenter uses available renewable energy as much as possible and then uses brown energy to satisfy the rest of the datacenter energy demand) for several months to generate historical running data to creating and training its own MARL model. The prediction model is trained by the historical data and then used to predict its resource demand. Other existing datacenters still use their own MARL agent models to make decisions and keep updating their own MARL models. In the next section, we propose a method to handle the shortage of renewable energy in such a case.

\subsection{Deadline-Guaranteed Job Postponement}

Because of many factors such as weather, the amount of generated renewable energy of a generator is not stable and may deviate a lot from the predicted amount. For example, a storm may limit the amount of solar energy supply or the wind energy generator cannot work during extreme high wind-speed situation. When the actually generated energy amount is less than the total requested amount, as mentioned above, a generator then distributes the generated energy to the requesters proportionally based on their requested amounts. In this case, a datacenter receives an amount less than its requested amount, which may lead to job SLO violations due to insufficient renewable energy and switching to brown energy. A problem here is how can we avoid the SLO violations and also reduce the monetary cost caused by brown energy usage? To handle this problem, we propose a Deadline-Guaranteed Job Postponement (DGJP) method. Compared with the previous works which don't consider the power outage in job scheduling, DGJP postpones the execution of the jobs with longer deadline requirements. That is, this method moves the unurgent jobs from the period with insufficient energy supply to the period with extra energy supply while still providing deadline guarantee.

We define a job's urgency coefficient as the time it must continue to run in order to meet its deadline requirement; that is, the difference between the deadline and the estimated job remaining running time [34]. For example, job 1 has deadline in 60 minutes and its remaining running time is 10 minutes and job 2 has deadline in 30 minutes and the remaining running time is 25 minutes. Then, job 1's urgency coefficient is $60-10=50$ minutes, i.e., it must continue to run no later than in 50 minutes, and job 2's urgency coefficient is $30-25=5$ minutes, i.e., it must continue to run no later than in 5 minutes. Then, job 1 has lower urgency than job 2, and 
job 1 should be paused instead of job 2. We can estimate the energy consumption of a job's remaining running based on the amount of the computing resource assigned to the job [23]. When the renewable energy outage happens, DGJP first sorts the running jobs based on the descending order of the urgency coefficients and sums the energy demand of the job from the top one by one until the sum is no less than the energy shortage in this time slot. Then, the selected running jobs will be paused and put in the pause job queue.

The jobs in the queue are sorted in the ascending order of the urgency coefficients. A paused jobs will resume when it is its urgency time or when there is an extra renewable energy supply in the datacenter whichever happens earlier. The latter occurs in two cases. First, we assume that a generator will compensate the deficiency amount when the actually generated amount is higher than the total requested amount. Second, a datacenter's actual demand is less than the predicted demand. When there is extra energy supply, the jobs in the queue will be selected to run from the top one by until the queue is empty or the selected jobs consume all the extra energy in this time slot. Note that the selected jobs may compete with other running jobs on the computing resources. We rely on the job scheduler to reschedule the selected paused jobs to avoid the computing resource competition.

\section{PERFORMANCE EVALUATION}

\subsection{Experiment Settings}

In our experiment, we use the trace data and approaches introduced in Section 3.1 to conduct trace-driven experiments. We randomly assigned 30 million Wikipedia web pages into one datacenter and consider one request as one job. The newly scheduled jobs will arrive on a datacenter by every hour following the request arrival rate in the Wikipedia trace data. We generate 30 to 150 datacenters in our experiment and the default number of datacenters is 90 . We assigned each job a deadline $x$ randomly chosen from the range of $[1,5]$ time slots (i.e., hours). We generated 60 renewable energy generators, and half of them are solar energy generators and the rest are wind energy generators. We assume that the renewable energy generators are evenly distributed in Virginia, California, and Arizona. To simulate the amount of generated energy in each generator, we used the product of the energy amount from the trace and a stochastic coefficient randomly chosen from range $[1$, 10]. For example, the amount of generated energy from one solar energy generator is the calculated solar energy amount from the trace times the stochastic coefficient. We set $\alpha_{1}=0.3, \alpha_{2}=0.25$, and $\alpha_{3}=0.45$ since this setting achieve the best performance in our experiments. For the five-year long datasets, the training time (for SARIMA prediction models and MARL model) is three years and the testing or simulation time period is two years. A datacenter switches to the brown energy supply upon renewable energy shortage.

\subsection{Comparison Methods}

As there are no previous works for the research problem in this paper, we developed several comparison methods by referring to previous works as described in Section 2.

(1) GS. This method is developed by referring to the "green scheduling" for one datacenter [32], which tends to run more jobs when the energy supply is higher. In GS, a datacenter uses FFT to predict the amount of generated energy at each time slot in the next month and sends its demand to the generator with the highest predicted generated energy amount (the sum of the series of predicted values). The energy demand of each job is calculated based on the computing resource assigned on this job by the job scheduler. A generator conducts energy allocation among multiple requesters and notifies them. If the datacenter cannot receive the demanded amount from the generator, it requests the remaining demand from the next generator with the highest predicted generated energy amount. This process repeats until the datacenter's total demand is satisfied.

(2) REM (Renewable Energy Management). REM is developed by referring to a job scheduler based method for one datacenter [22], which aims to minimize the monetary cost of energy. REM is similar as the above method except that a datacenter uses our method for prediction and chooses the generator with the lowest average unit price over one month to request first in order to minimize the monetary cost.

(3) REA. This method is developed by referring to the Renewable Energy-Aware reinforcement learning [48]. This method is the same as GS except when a datacenter does not receive sufficient renewable energy, it uses RL to determine which jobs need to be postponed to the next time slot (i.e., next hour) in every hour. The inputs of $\mathrm{RL}$ include deadline and energy demand of each job, the output is the datacenter to run each job.

(4) SRL (Single Reinforcement Learning). This method is developed by referring to the method that enables a single datacenter to determine which uses RL for a single datacenter renewable energy management [21]. In this method, each datacenter uses LSTM ML technique to predict the amount of each generator's generated energy and uses RL to determine the amount of energy requested from one datacenter to which energy generators in order to minimize the monetary cost and carbon emission with lower SLO violation. However, since there is competition among all the datacenters for energy requests, each datacenter may not be able to achieve its goals easily and they also need several times to achieve the enough energy so that the energy price will be increased.

It is noteworthy that the effectiveness of each component in MARL can be seen from the performance difference of different comparison methods. First, GS uses FFT for energy generation and demand prediction while REM uses the same prediction method (ARIMA) used in MARL, so the performance difference between GS and REM shows the performance improvement obtained by the prediction method in MARL. Specifically, REM improves GS by $1 \%$, $10 \%$, and $9 \%$ in the SLO satisfaction ratio, monetary cost and carbon emission respectively. Second, SRL uses single RL method without considering agent competition and MARL uses the multi-agent $\mathrm{RL}$ method considering agent competition, so the performance difference between SRL and MARLw/oD shows the performance improvement obtained by the multi-agent RL. Specifically, MARLw/oD improves SRL by $20 \%, 13 \%$, and $10 \%$ in the SLO satisfaction ratio, monetary cost and carbon emission respectively.The performance improvement obtained from the deadline-guaranteed job postponement is shown by the difference between MARLw/oD and MARL. Specially, MARL improves MARLw/oD by $3 \%, 5 \%$, and $4 \%$ in the SLO satisfaction ratio, monetary cost and carbon emission respectively. 


\subsection{Performance Metrics}

- Prediction accuracy. It is explained in Section 3.1.

- SLO satisfaction ratio. It is defined as the percentage of jobs whose deadlines are satisfied in a datacenter during the testing time. If a job cannot be completed by its deadline due to insufficient renewable energy before switching to the brown energy supply upon renewable energy shortage, we consider that its SLO is not satisfied. - Monetary cost and carbon emission. We calculate the total monetary cost and carbon emission based on the calculation method introduced in [21] and the real price dataset $[8,12,13]$ during the testing time. The price dataset contains the price of brown energy and renewable energy electricity price respectively at each hour. In general, the price range for the solar energy is $[50,150] \mathrm{USD} / \mathrm{MWh}$, for the wind energy is $[30,120] \mathrm{USD} / \mathrm{MWh}$, and for the brown energy is $[150,250] \mathrm{USD} / \mathrm{MWh}$.

- Time overhead. It is the average time latency for computing the decisions for the datacenter-generator matching problem. Since the ML training is offline, it is not time sensitive.

\subsection{Experimental Results}

Figure 8 shows the predicted and actual amount of renewable energy and the prediction accuracy for the solar energy and wind energy on one randomly selected solar generator and one randomly
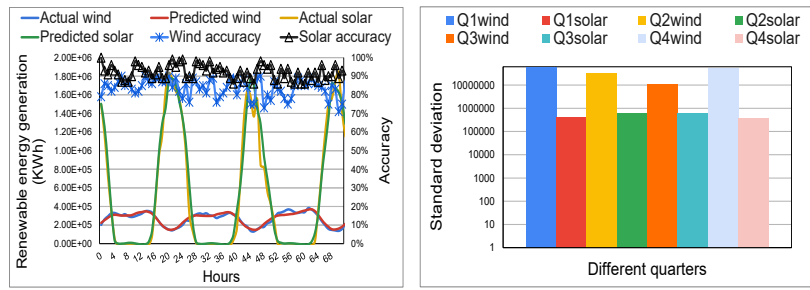

Figure 8: Renewable energy Figure 9: Standard deviation generation and prediction. for different seasons.

selected wind energy generator using ARIMA in three continuous days selected from the dataset. We see that the renewable energy generation follows a one-day periodical pattern and most of the predicted values and the actual value are very close. The accuracy for solar energy is above $90 \%$ all the time and above $70 \%$ for wind all the time. The result also shows that the accuracy of the solar energy prediction is higher than the wind energy prediction. From the actual solar energy values, we observed that the solar energy is more seasonal, so it can be predicted with a higher accuracy than the wind energy. Figure 9 shows the average standard deviation of the amount of solar energy and wind energy in two years where Q1 means the first quarter in a year. The standard deviation of the wind energy is over 1000 times of the standard deviation of the solar energy for the four quarters. The results show that the solar energy is more stable compared with wind energy in different seasons. In another word, solar energy is more stable and predictable to be a more reliable type of renewable energy.

Figure 10 and 11 show the energy consumption for a single datacenter randomly selected from the generated datacenters and all the 90 datacenters from March 1, 2015 to May 31, 2015. From both figures, we can observe that the energy consumption exhibits periodical patterns (in 7 days) during a certain time period, which makes it feasible to conduct energy demand prediction.
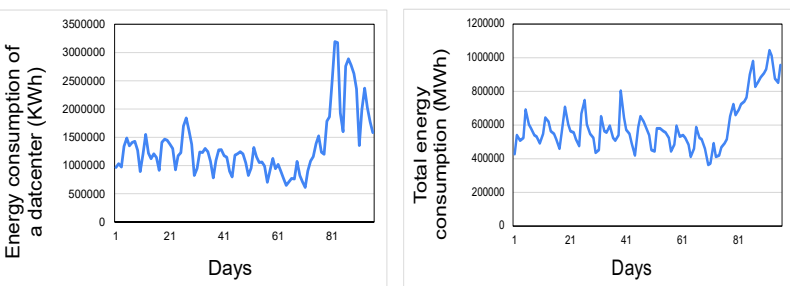

Figure 10: Energy consump-Figure 11: Energy consumption for one datacenter. tion for all the datacenters.

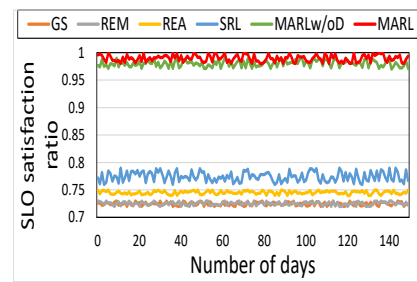

Figure 12: SLO satisfaction ratio for each day.

Figure 12 shows the SLO satisfaction ratio versus the number of days (in the first six months) in time series. We use MARLw/oD to denote MARL without DGJP. The result follows MARL $>$ MARLw/oD $>$ SRL $>$ REA $>$ REM $\approx$ GS. MARL and MARLw/oD achieve over $97 \%$ and $96 \%$ SLO satisfaction ratio, respectively. The ratio for SRL, REA, REM, and GS is around 76\%, 75\%, 72\%, and $72 \%$, respectively. MARL improves the comparison methods up to $35 \%$. Both REM and GS use the FFT pattern based prediction method to predict the amount of renewable energy generation. Compared with ARIMA, the lower prediction accuracy may mislead the energy assignment and then makes REM and GS unable to use renewable energy to fulfill the energy demand all the time, resulting in lower SLO satisfaction ratio. REA uses the same prediction model as in GS and uses RL to do the job scheduling, which can achieve much better decisions on job scheduling since RL can react to the poor job scheduling decisions.

SRL uses LSTM to do the workload and energy generation in long term prediction and uses RL in one datacenter to determine the how much energy should request to which energy generators for one month. Achieving lower SLO violation is one of its goals. Also, its higher prediction accuracy than FFT leads to higher SLO satisfaction ratio compared with REA. However, the RL agents on different datacenters do not consider the competition between the datacenters so that some of the datacenter cannot receive enough energy. MARL and MARLw/oD implement an MARL agent on each datacenter and use SARIMA for predicting energy demand and energy consumption in long term. SARIMA has higher accuracy than LSTM in the prediction. Thus, MARL and MARLw/oD can generate better datacenter-generator matching plan based on more accurate prediction, resulting in lower possibility that the renewable energy cannot fulfill the demand of one datacenter. Meanwhile, using the MARL algorithm, in MARL and MARLw/oD, the RL agent considers the competition among agents and makes decision on the datacentergeneration matching plan to always fulfill energy demand for its datacenter regardless of other competitors' actions. In addition, MARL has the DGJP algorithm that postpone running some jobs from the time with insufficient renewable energy supply to the time with extra renewable energy supply while still guaranteeing the jobs' deadlines. Therefore, MARL and MARLw/oD can always 
achieve the highest SLO performance ratios than other methods. MARLw/oD does not postpone running some jobs when the energy supply is not enough so that some jobs may not be completed before their deadlines because of energy shortage. Therefore, MARLw/oD achieves lower SLO satisfaction ratio than that of MARL.
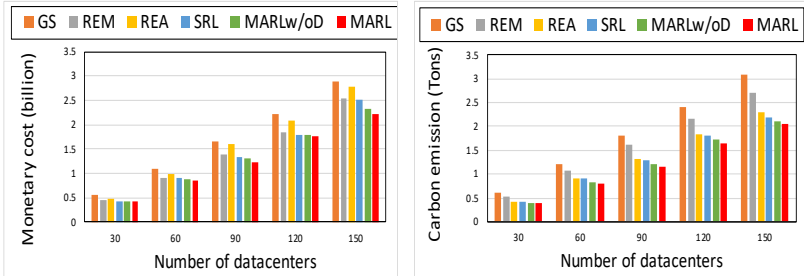

Figure 13: Total money cost. Figure 14: total carbon emission.

Figure 13 shows the total monetary cost of each method versus the number of datacenters. The results follow MARL $<$ MARLw/oD $<$ $\mathrm{SRL}<\mathrm{REM}<\mathrm{REA}<\mathrm{GS}$. In default 90 datacenters, the monetary cost of MARL and MARLw/oD is 1.25 billion and 1.29 billion, respectively. The monetary cost of SRL, REA, REM, and GS is 1.32 billion, 1.41 billion, 1.54 billion and 1.58 billion, respectively. MARL reduces the monetary cost of the comparison methods by up to $19 \%$ and 0.33 billion dollars in 90 days. GS and REA do not take the monetary cost into account so that these two methods generate higher monetary cost than others. Meanwhile, the lower SLO satisfaction ratio caused by lower energy generation prediction accuracy means that these two methods use more brown energy to fulfill the energy demand, which increases the monetary cost due to higher unit price of brown energy. Thus, GS and REA generate the highest monetary cost. Since REA has an RL algorithm to postpone jobs when the datacenter does not receive sufficient renewable energy, it reduces the brown energy consumption. Therefore, REA has lower monetary cost than GS. We also see that REA generates lower monetary cost than GS and REM. This is because REM aims to reduce the total monetary cost while trying to use renewable energy as much as possible. Also, its lower SLO satisfaction ratio than GS means it uses less brown energy than GS.

Minimizing monetary cost is one of SRL's goals. Due to the reasons explained for Figure 12, SRL can make better datacentergenerator matching plan to provide sufficient renewable energy supply, which helps reduce brown energy usage due to renewable energy shortage. This helps reduce the monetary cost. As a result, SRL generates less monetary cost than REM, REA and GS. Due to the reasons explained for Figure 12, MARL and MARLw/oD make better datacenter-generator matching plan than SRL that reduces the times of the renewable energy shortage.

Figure 14 shows the carbon emission of each method versus the number of datacenters. The results follow $M A R L \approx M A R L w / o D<S R L$ $<$ REA $<$ REM $<$ GS. The carbon emission of MARL and MARLw/oD is 1.21 tons and 1.23 tons, respectively. The carbon emission of GS, REM, REA, and SRL is 1.8 tons, 1.6 tons, 1.31 tons, and 1.27 tons, respectively. MARL and MARLw/oD reduce the carbon emission by up to $33 \%$ and 0.59 tons carbon emission for 90 days. GS has the largest carbon emission since GS uses FFT energy prediction which has lower accuracy. The less accurate energy prediction leads to less efficient datacenter-generator matching plan which needs

more brown energy resulting in the highest carbon emission. Since renewable energy is cheaper than brown energy, even with the same prediction method, REM aims to reduce the monetary cost while tries to use more renewable energy, that leads to use more renewable energy than GS and then less carbon emission. REA has an RL to determine which jobs to postpone to the next time slot, so it uses less brown energy and hence less carbon emission.

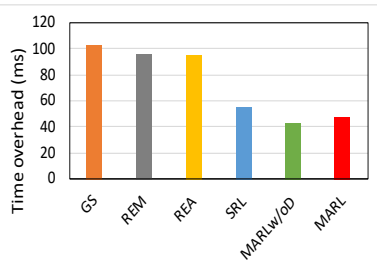

Figure 15: Average time overhead.

Figure 15 shows the average time overhead for one datacenter's demand-supply match plan generation of each method. The time overhead is measured only for the decision making time without considering the RL based method training time. The results follows MARLw/oD $<$ MARL $<$ SRL $<$ GS $\approx$ REM $\approx$ REA. The time overhead of GS, REM, REA, SRL, MARLw/oD and MARL is $102 \mathrm{~ms}, 95 \mathrm{~ms}, 94 \mathrm{~ms}$, $53 \mathrm{~ms}, 43 \mathrm{~ms}$ and $48 \mathrm{~ms}$, respectively. GS, REM and REA need more time to find the energy generator that can fulfill their energy demand because they do not consider the long-term planning or the energy competition. MARL and MARLw/oD use multi-agent RL, which considers the energy competition so that these two methods use less number of energy requests to generate the datacentergenerator matching plan and then less time overhead in decision making. The overhead of MARL is a little larger than MARLw/oD since MARL needs to execute DGJP which introduces extra time overhead. Therefore, MARL and MARLw/oD can achieve the better time overhead performance.

Figure 16 shows the average SLO satisfaction ratio versus different number of datacenters during two years. The results follow MARL $>$ MARLw/oD $>$ SRL $>$ REA $>$ GS $\approx$ REM due to the same reason in Figure 12. It is noteworthy that MARL can always achieve the best SLO satisfaction ratio over $98 \%$ with the increasing number of datacenters, which indicates that it has high scalability.

\section{CONCLUSIONS AND FUTURE WORK}

Nowadays, the energy consumption of cloud datacenters has been rapidly increasing. Because of the negative effect of the traditional brown energy on the environment due to carbon emission and high monetary cost, more and more datacenters resort to renewable energy as the energy supply. However, the instability of renewable energy may lead to power shortage in the datacenter and lead to job SLO violation. Also, datacenters belong to different cloud service providers do not share information and hence cannot coordinate in renewable energy requesting to the generators. As a result, it is necessary to find a datacenter-generator matching plan which can fully utilize the renewable energy while satisfy the energy demand of each datacenter and minimize the monetary cost of each datacenter. In this paper, we propose a multi agent RL based method (MARL) that deploys an RL agent on each datacenter to make the energy request decisions without the information sharing between all the 
datacenters aiming to achieve higher SLO satisfaction ratio, lower monetary cost and lower carbon emission. Through comparing the prediction accuracy performance of several typical ML techniques to make the long-term prediction for the energy demand and the energy generation based on real traces, we identify ARIMA to be used in MARL for long-term prediction for the energy demand of datacenter and the energy generation of renewable generator We also propose deadline-guaranteed job postponement method (DGJP) to postpone unurgent jobs with later SLO deadline requirements to the time period with higher amount of renewable energy supply. The comprehensive trace-driven experiments show that MARL has superior performance compared with other methods in SLO satisfaction ratio with less monetary cost and carbon emission.

In the future work, we will investigate how to jointly conduct workload balance considering the job computing resource competition and how to distribute the generated energy to datacenters.

\section{ACKNOWLEDGMENTS}

This research was supported in part by U.S. NSF grants NSF-1827674, CCF-1822965, FHWA grant 693JJ31950016, Microsoft Research Faculty Fellowship 8300751, and AWS Machine Learning Research Awards. We would like to thank Prof. Rajkumar Buyya's help on this paper.

\section{REFERENCES}

[1] [Accessed in Apr. 2021]. Amazon has a cost-cutting plan for the boom-and-bust oil business, as rival tech giants target energy industry. In https://www.cnbc.com/2019/03/14/amazon-has-a-cost-cutting-plan-for-the-boomand-bust-oil-business.html.

[2] [Accessed in Apr. 2021]. Announcing round-the-clock clean energy for cloud. In https://cloud.google.com/blog/topics/inside-google-cloud/announcing-round-theclock-clean-energy-for-cloud.

[3] [Accessed in Apr. 2021]. AWS Sustainability in the Cloud. In https://sustainability.aboutamazon.com/environment/the-cloud?energyType=true.

[4] [Accessed in Apr. 2021]. Consequences of Carbon Emissions for $\mathrm{Hu}$ mans. In https://sciencing.com/consequences-of-carbon-emissions-for-humans12730960.html.

[5] [Accessed in Apr. 2021]. Electricity rates by state. In https://www.chooseenergy.com/electricity-rates-by-state/.

[6] [Accessed in Apr. 2021]. How to Improve Data Center Power Consumption and Energy Efficiency. In https://www.vxchnge.com/blog/power-hungry-the-growingenergy-demands-of-data-centers.

[7] [Accessed in Apr. 2021]. Making data centers part of Europe's sustainable energy transformation. In https://blogs.microsoft.com/eupolicy/2019/06/18/data-centerssustainable-energy-transformation/.

[8] [Accessed in Apr. 2021]. Measurement and instrumentation data center. In https://midcdmz.nrel.gov/l.

[9] [Accessed in Apr. 2021]. NREL Solar Radiation Research Laboratory-Solar Dataset. In https://midcdmz.nrel.gov/apps/sitehome.pl?site $=B M S$.

[10] [Accessed in Apr. 2021]. NREL Wind Technology Center-Wind Dataset. In https://midcdmz.nrel.gov/apps/sitehome.pl?site $=N W T C$.

[11] [Accessed in Apr. 2021]. Study: Data Centers Responsible for 1 Percent of All Electricity Consumed Worldwide. In https://www.datacenterknowledge.com/energy/study-data-centers-responsible-1percent-all-electricity-consumed-worldwide.

[12] [Accessed in Apr. 2021]. Which is the Cheapest Renewable Energy Supplier in 2019. In https://theswitch.co.uk/blog/energy/cheapest-green-supplier.

[13] [Accessed in Apr. 2021]. Wholesale Electricity and Natural Gas Market Data. In https://www.eia.gov/electricity/wholesale/.

[14] [Accessed in Apr. 2021]. Wikistats pageview files. In https://dumps.wikimedia.org/other/pagecounts-ez/.

[15] D. Borthakur, J. Gray, J. Sarma, K. Muthukkaruppan, N. Spiegelberg, H. Kuang, K. Ranganathan, D. Molkov, A. Menon, and S. Rash. 2011. Apache Hadoop goes realtime at Facebook. In Proc. of SIGMOD.

[16] L. Buşoniu, R. Babuška, and B. De Schutter. 2010. Multi-agent reinforcement learning: An overview. In Innovations in multi-agent systems and applications.

[17] R. J. Chansler. 2012. Data availability and durability with the hadoop distributed file system. In Proc. of USENIX.
[18] P. Chen, A. Niu, D. Liu, W. Jiang, and B. Ma. 2018. Time series forecasting of temperatures using SARIMA: An example from Nanjing. In Proc. of IOP.

[19] M. Dayarathna, Y. Wen, and R. Fan. 2015. Data center energy consumption modeling: A survey. IEEE Communications Surveys and Tutorials (2015).

[20] I. De Courchelle and T. Guérout. 2019. Green energy efficient scheduling management. Simulation Modelling Practice and Theory (2019).

[21] J. Gao, H. Wang, and H Shen. 2020. Smartly Handling Renewable Energy Instability in Supporting A Cloud Datacenter.. In Proc. of IPDPS.

[22] I. Goiri, M. Haque, K. Le, R. Beauchea, T. Nguyen, J. Guitart, J. Torres, and R. Bianchini. 2019. Matching renewable energy supply and demand in green datacenters. Ad Hoc Networks (2019).

[23] C. Gu, C. Liu, J. Zhang, H. Huang, and X. Jia. 2015. Green scheduling for cloud data centers using renewable resources. In Proc. of INFOCOM WKSHPS.

[24] L. Gu, J. Cai, D. Zeng, Y. Zhang, H. Jin, and W. Dai. 2019. Energy efficient task allocation and energy scheduling in green energy powered edge computing. Future Generation Computer Systems (2019).

[25] Yuxiu Hua, Zhifeng Zhao, Rongpeng Li, Xianfu Chen, Zhiming Liu, and Honggang Zhang. 2019. Deep learning with long short-term memory for time series prediction. IEEE Communications Magazine (2019).

[26] M. Lanctot, V. Zambaldi, A. Gruslys, A. Lazaridou, K. Tuyls, J. Pérolat, D. Silver, and T. Graepel. 2017. A unified game-theoretic approach to multiagent reinforcement learning. In Advances in neural information processing systems.

[27] H. Lei, R. Wang, T. Zhang, Y. Liu, and Y. Zha. 2016. A multi-objective coevolutionary algorithm for energy-efficient scheduling on a green data center. Computers \& Operations Research (2016).

[28] J. Li, Z. Li, K. Ren, and X. Liu. 2011. Towards optimal electric demand management for internet data centers. Trans. on Smart Grid (2011).

[29] Y. Li and X. Wang. 2019. Thermal-aware hybrid workload management in a green datacenter towards renewable energy utilization. Energies (2019).

[30] M. Littman. 2001. Value-function reinforcement learning in Markov games. Cognitive systems research (2001).

[31] Michael L Littman. 1994. Markov games as a framework for multi-agent reinforcement learning. In Proc. of Machine learning.

[32] Z. Liu, Y. Chen, C. Bash, A. Wierman, D. Gmach, Z. Wang, M. Marwah, and C. Hyser. 2012. Renewable and cooling aware workload management for sustainable data centers. In Proc. of ACM SIGMETRICS.

[33] F. Mantovani and E. Calore. 2018. Performance and power analysis of hpc workloads on heterogeneous multi-node clusters. Journal of Low Power Electronics and Applications (2018).

[34] K. Morton, A. Friesen, M. Balazinska, and D. Grossman. 2010. Estimating the progress of MapReduce pipelines. In Proc. of ICDE.

[35] J. Pierson, G. Baudic, S. Caux, and B. Celik. 2019. Datazero: Datacenter with zero emission and robust management using renewable energy. IEEE Access (2019).

[36] L. Rao, X. Liu, L. Xie, and W. Liu. 2010. Minimizing electricity cost: optimization of distributed internet data centers in a multi-electricity-market environment. In Proc. of INFOCOM.

[37] C. Ren, D. Wang, B. Urgaonkar, and A. Sivasubramaniam. 2012. Carbon-aware energy capacity planning for datacenters. In Proc. of MASCOTS

[38] N. Sapankevych and R. Sankar. 2009. Time series prediction using support vector machines: a survey. IEEE Computational Intelligence Magazine (2009).

[39] K. Shvachko, H. Kuang, S. Radia, and R. Chansler. 2010. The hadoop distributed file system. In Proc. of MSST.

[40] C. Stewart and K. Shen. 2009. Some joules are more precious than others: Managing renewable energy in the datacenter. In Proc. of HotPower.

[41] O. Vinyals, I. Babuschkin, W. Czarnecki, M. Mathieu, A. Dudzik, J. Chung, D. Choi, R. Powell, T. Ewalds, and P. Georgiev. 2019. Grandmaster level in StarCraft II using multi-agent reinforcement learning. Nature (2019).

[42] H. Wang, Z. Liu, and H. Shen. 2020. Job scheduling for large-scale machine learning clusters. In Proc. of CoNEXT.

[43] H. Wang, H. Shen, and Z. Li. 2018. Approaches for resilience against cascading failures in cloud datacenters. In Proc. of ICDCS.

[44] H. Wang, H. Shen, Q. Liu, K. Zheng, and J. Xu. 2020. A Reinforcement Learning Based System for Minimizing Cloud Storage Service Cost. In Proc. of ICPP.

[45] H. Wang, H. Shen, C. Reiss, A. Jain, and Y. Zhang. 2020. Improved Intermediate Data Management for MapReduce Frameworks. In Proc. of IPDPS.

[46] C. Watkins and P. Dayan. 1992. Q-learning. Machine learning (1992).

[47] M. Webb. 2008. SMART 2020: enabling the low carbon economy in the information age, a report by The Climate Group on behalf of the Global eSustainability Initiative (GeSI). Creative Commons (2008).

[48] C. Xu, K. Wang, P. Li, R. Xia, S. Guo, and M. Guo. 2018. Renewable energy-aware big data analytics in geo-distributed data centers with reinforcement learning. Trans. on NSE (2018).

[49] X. Yang, Y. Wang, and H. He. 2019. Deep Reinforcement Learning for Economic Energy Scheduling in Data Center Microgrids. In Trans. on PESGM.

[50] D. Yi, X. Zhou, Y. Wen, and R. Tan. 2019. Toward efficient compute-intensive job allocation for green data centers: A deep reinforcement learning approach. In Proc. of ICDCS. 